\title{
FINITE ELEMENT MODELING OF INTERMUSCULAR INTERACTIONS AND MYOFASCIAL FORCE TRANSMISSION
}

\author{
Can A. Yucesoy ${ }^{1}$, Bart F.J.M. Koopman ${ }^{1}$, Peter A. Huijing ${ }^{1,2}$, Henk J. Grootenboer ${ }^{1}$ \\ 'Integrated Biomedical Engineering for Restoration of Human Function, Department of Mechanical Engineering, \\ University of Twente, Enschede, The Netherlands \\ ${ }^{2}$ Instituut voor Fundamentele en Klinische Bewegingswetenschappen, Faculteit Bewegingswetenschappen, \\ Vrije Universiteit, Amsterdam, The Netherlands
}

\begin{abstract}
A finite element muscle model to study the principles of intermuscular myofascial force transmission is developed. The results obtained explain force differences at the distal and proximal tendons of muscles that have mechanical interaction. This is in agreement with experimental findings in other recent studies. The strain distributions found along the fiber direction indicate intermuscular myofascial force transmission. A consequence is that active force generated within one muscle may be exerted at the tendon of another muscle.

Keywords- Finite element method, Intermuscular interactions, Myofascial force transmission, Rat extensor digitorium longus
\end{abstract} (EDL) muscle

\section{INTRODUCTION}

Performing the complex task of controlled bodily movements requires the transmission of the force generated by the skeletal muscles onto the skeleton. Although for this transmission, the myotendinous junction is widely accepted to be the major site, rather recently the transmission from the muscle fibers onto the intramuscular connective tissue has been proven to be important as well [1-5]. Referring to this kind of transmission as myofascial force transmission, Huijing [6] suggested that force could be transmitted from the muscle by this path as well (extramuscular myofascial force transmission). Recent experiments revealed significant changes in the length-force characteristics of rat extensor digitorium longus (EDL) muscle on interference with [7] or interactions with the surrounding extramuscular tissues. It was also shown that adjacent muscles interact mechanically [8]. In the present work, the two-domain finite element muscle model described recently [9] is extended to include the principles of intermuscular myofascial force transmission. In experimental work on the subject, the muscle is not accessible for local strain measurements, as it has to remain surrounded by compartmental connective tissues. This modeling study aims at providing such analysis of local strain to enhance understanding of this concept.

\section{METHODOLOGY}

A 3D-finite element muscle model (linked fiber-matrix mesh model: lfmm model) with a two domain approach was developed [9]. This model consists of two meshes that are linked elastically representing the extracellular matrix (matrix mesh) and muscle fiber domains (fiber mesh), which occupy the same space. These two meshes are built using earlier developed myofiber or extracellular matrix elements that are introduced into the finite element program ANSYS 5.5.1 as user defined elements. The two meshes are rigidly connected to single layers of elements representing the muscles' aponeurosis, for which a standard element, HYPER58 from the element library of ANSYS 5.5.1 is used.

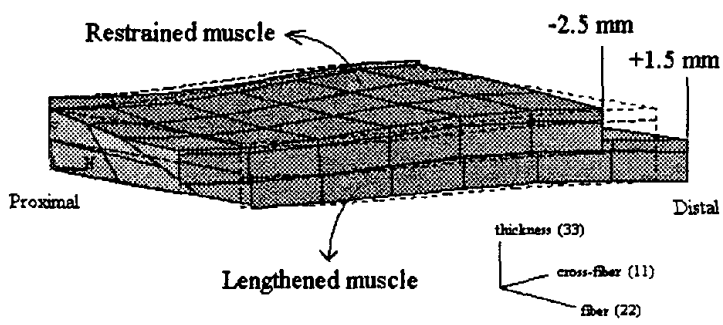

Fig.1. The deformed shape of the model. The model consists of two EDL models. One that is restrained at a low length (i.e. $2.5 \mathrm{~mm}$ shorter than the initial length), which has interaction with another EDL model that is lengthened distally (from $\Delta / m=-2.5 \mathrm{~mm}$ to $\Delta l m=1.5 \mathrm{~mm}$ ). Muscular geometry at the initial muscle length $(\mathrm{lm}=28.7 \mathrm{~mm})$ is represented by dotted lines. A 3D local coordinate system is used for the analysis and presentation of the results.

In the present study, using the same methods, a model representing whole EDL isolated from the surrounding connective tissues was built. As a subsequent step, two of such models were connected: the corresponding nodes of the matrix meshes of the two models were linked elastically. Such links represent the intermuscular connections, providing a means to show the principles of mechanical interaction between the two muscles and a pathway for intermuscular force transmission.

To assess the principles of such interaction and force transmission, one EDL model referred to as restrained muscle was kept at a low length $(\Delta l m=2.5 \mathrm{~mm}$ below the original length), while the other model (referred to as lengthened muscle) was lengthened from this low length up to $1.5 \mathrm{~mm}$ over the original length (Fig. 1). Throughout the analysis, both modeled muscles were maintained maximally activated. The length-force characteristics of the isolated model will be compared to experimental data for EDL in such conditions. Stress and strain distributions of the isolated model and the model with intermuscular links will be compared as well.

\section{RESULTS}

Isolated EDL Model

The length- total force characteristics of modeled isolated EDL and experimental data [7] are shown in Fig. 2. The model forces are maximally $5 \%$ above or below the experimental ones suggesting a fairly good agreement. For the highest lengths considered, the model forces remain high whereas the experimental force decreases with increasing length. This could be due to over estimated passive length-force characteristics of the model as well as the, unrealistic modeling assumption of an initially uniform sarcomere distribution. 


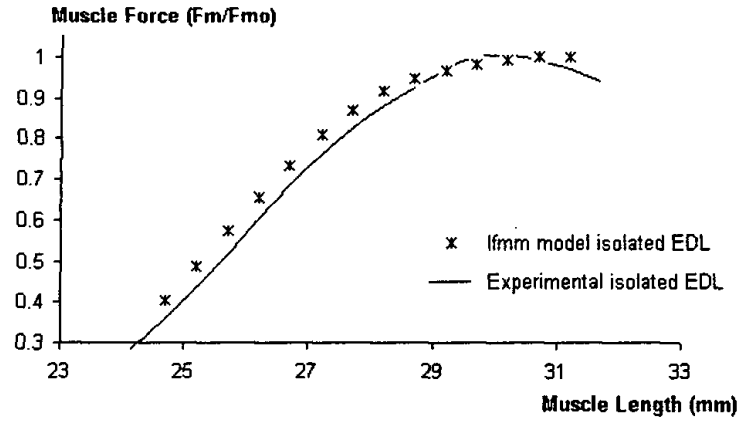

Fig.2. The isometric muscle length-total force curve for isolated rat EDL muscle. Experimental EDL data is adopted from [7]. The muscle force (Fm) is normalized for optimum force (Fmo). Initial muscle length equals $28.7 \mathrm{~mm}$.

\section{Model with Intermuscular Connections}

The muscle length-total force characteristics of the lengthened and the restrained muscle, which have intermuscular links between their matrix meshes representing intramuscular collagen is presented in Fig. 3. Note that the distributions of strain and stress in the fiber mesh of the restrained muscle are provided for three lengths of the lengthened muscle (Fig. 4). At $\Delta l m=-2.5 \mathrm{~mm}$ (i.e. both

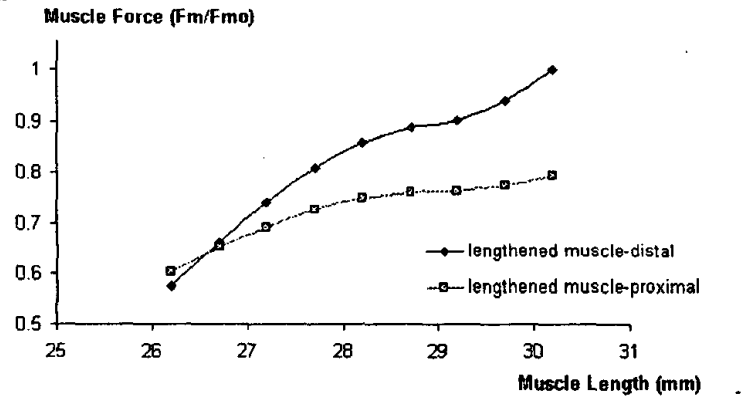

b

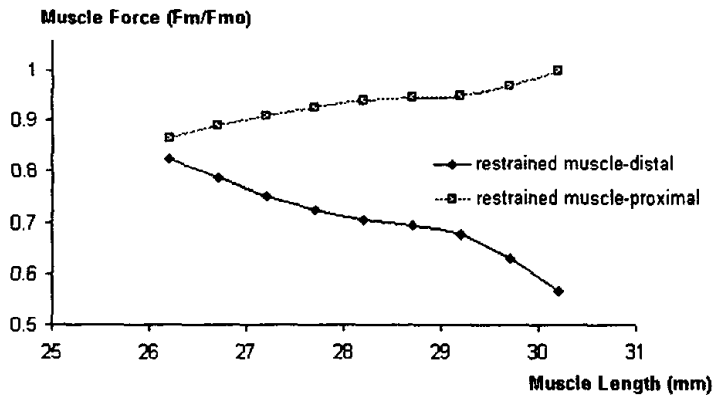

Fig.3. Comparison of proximal and distal forces of the modeled EDL muscles. (a) Length-total force curves for the lengthened muscle. The muscle force $(\mathrm{Fm})$ is normalized with respect to optimum distal force (Fmo).

(b) Total force for the restrained muscle as a function of the lengthened muscles' length. The muscle force is normalized for optimum proximal force. Initial muscle length equals $28.7 \mathrm{~mm}$ muscles are at the lowest length), the fiber direction strain $\left(\varepsilon_{22}\right)$ does not exhibit variations, i.e. the lengths of sarcomeres in series within fibers are highly uniform (Fig. 4a). As the lengthened muscle reaches higher lengths $(\Delta / m=+1.5 \mathrm{~mm})$ this homogeneity is replaced by on average $10 \%$ longer sarcomeres at the distal end of muscle fibers than at their proximal end.

The consequences of these differences in strain are clearly visible on the fiber direction stresses $\left(\sigma_{22}\right)$. If both muscles are at low length, the stresses along the fibers of the restrained muscle are rather uniform. As the adjacent muscle is lengthened, distributions of $\sigma_{22}$ become apparent: at distal ends of fibers approximately $50 \%$ higher stresses are encounterd than their proximal ends.

For a further assessment of mechanics of interaction between the muscles, the fiber direction strains and stresses within the fiber mesh at the highest length studied (i.e. $\square l m=$ $+1.5 \mathrm{~mm}$ ) are considered for the lengthened muscle of the modeled pair, in comparisson to the isolated EDL model (Fig. 5). A clear difference in strain distributions between the two muscles is visible (Fig. 5a). Except for the minor distributions in the most proximal part of the isolated muscle (which is ascribable to the asymmetric EDL muscle geometry), the strains are highly uniform over the whole fiber mesh. In contrast, for the lengthened muscle with intermuscular connections to the restrained muscle, a high level of variation in $\varepsilon_{22}$ distribution is seen: The sarcomeres along the proximal ends of the muscle fibres are approximately $20 \%$

a Restrained Muscle
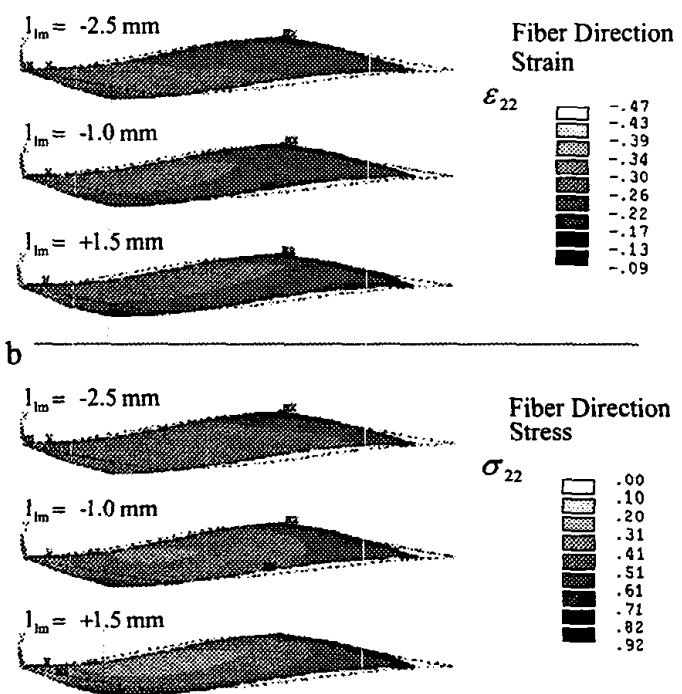

Fig.4. Distributions of fiber direction strain and stress for the fiber mesh of the restrained muscle as a function of the length of the lengthened muscle.

(a) Distributions of fiber strain at three muscle lengths.

(b) Distributions of fiber stress at three muscle lengths.

The dotted line contour indicates muscle geometry at the initial length. 


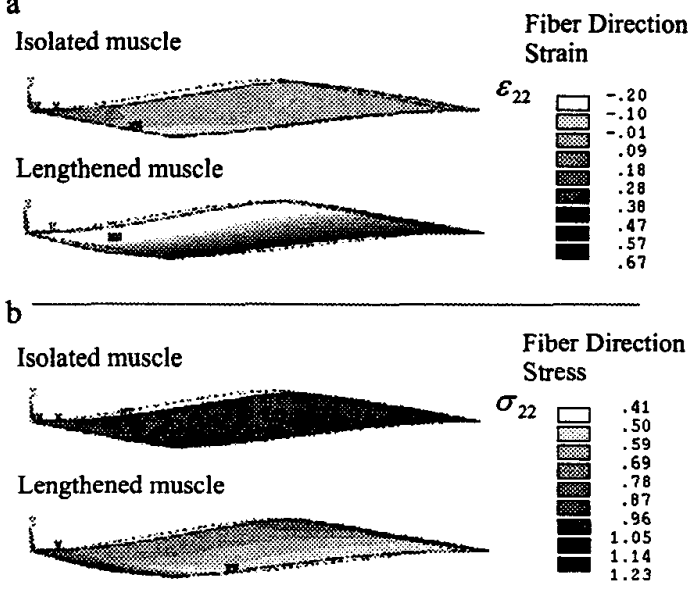

Fig.5. Comparison of strain and stress distributions for the fiber mesh of the lengthened and isolated muscles.

(a) Strain distributions of the fiber mesh for the fiber (22) direction.

(b) Stress distributions of the fiber mesh for the fiber (22) direction.

Both muscles are represented at the high length $(\Delta / m=+1.5 \mathrm{~mm})$. The dotted

line contour indicates muscle geometry at the initial length.

shortened, while towards the distal ends, their lengths gradually increase, such that the sarcomeres at most distal end are up to $67 \%$ longer. The differences between the muscles are also remarkable for the fiber direction stresses (Fig. 5b).In agreement with the strain distributions for the isolated muscle, the $\sigma_{22}$ values are highly uniform (i.e. approximating unity all over the fiber mesh). However, the lengthened muscle of the pair exhibits a sizable variation in $\sigma_{22}$ distribution, ranging from aproximately 0.80 to 0.50 . In that muscle stress decreases from the proximal end of the muscle fibres towards their distal ends. Also note that the mean stress for the isolated muscle (which is approximately unity) is significantly higher than that of the lengthened muscle with intermuscular

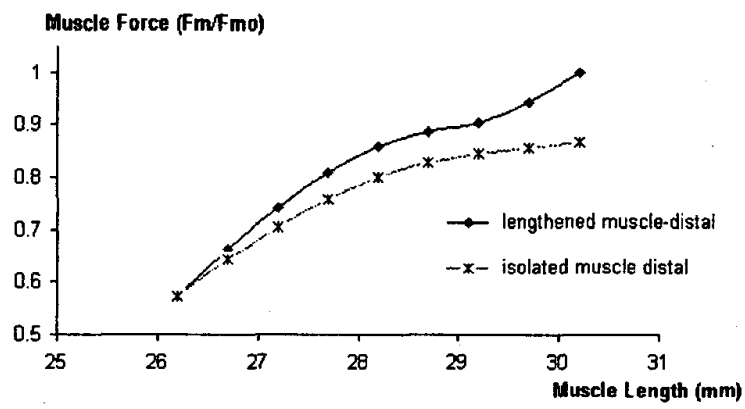

Fig.6. Comparison of length-total distal force curves of modeled muscle with intermuscular connections and isolated muscles.

Muscle force (Fm) is normalized for optimum force (Fmo) of the muscle with intermuscular connections. connections. Although $\sigma_{22}$ does attain values arround unity at certain limited locations, stress values over the major part of the fiber mesh are much lower. As fiber direction stress in the fiber mesh is the dominating factor determining muscle force, this result is highly interesting since the distal forces of the lengthened muscle are higher than that of the isolated muscle throughout the length range and at this particular length (Fig. 6). Such higher forces in spite of the lower mean stresses can be explained by intermuscular myofascial force transmission from the restrained muscle to the lengthened one.

\section{DISCUSSION}

Connective tissue network and force transmission

Muscle can be represented as an extensive 3D set of organized endomysial tunnels, within which the myofibers operate [3]. The identification of intramuscular force transmission from the lateral perimeter surface of myofibers onto the connective tissue network (myofascial force transmission) is relatively recent and limited. However, various studies indicated $[1,3]$ or experimentally confirmed $[1$, $3-6,10]$ such force transmission and suggested an important functional significance of the connective tissue network. This endomysial network is continuous with the perimysium system and can be regarded as a continuous system of tunnels or tubes as well. In addition, the muscular epimysium is continuous with this system as well as with the connective tissue of adjacent muscles in addition to other elements of the compartment in which the muscles operate [e.g. 5, 7]. Therefore, in vivo there is an additional route for force transmission out off the muscle, which completely bypasses the tendon of the muscle that was the source of the generated force $[5,7,11]$. In the majority of the earlier work on muscle functioning, muscles are investigated isolated from their surrounding [e.g. 1, 12, 13,14]. Such a type of experiment eliminates any possible mechanism of inter or extra-muscular force transmission from or to the muscle studied.

\section{Results of the model}

The model developed in this study provides the possibility to interpret effects of myofascial force transmission between two maximally activated muscles with a set of elastic links between their matrix meshes representing their intramuscular connections. A finite element muscle model with such an approach is novel. The remarkable result of the analysis is the significant differences between the distal and proximal forces of both of the restrained and lengthened muscles. These differences are such that for almost all of the length range considered, the distal forces of the lengthened muscle are higher than the proximal forces. This proximo-distal force difference increases with increasing muscle length. For the restrained muscle, the distal force decreases as the lengthened muscle reaches higher lengths. These results are highly compatible with the very recent findings in experimental work exploring intermuscular interaction via myofascial force transmission within rat hindlimb. In their study investigating the effects of blunt dissection and compartmental fasciatomy on rat EDL length-force characteristics, Huijing and Baan [7] showed that a systematic manipulation of the extra and intermuscular connective tissue structures altered proximal EDL force remarkably. These changes were decreases in force and 
changes in the muscle optimum length, as a result of decreased inter and extra-muscular interactions as EDL was dissected further. This indicates the importance of extramuscular connective tissue in force transmission.

The advantage of the present model is its capability to examine the mechanics by analysis of local stress and strain distributions. Such distributions reveal that even though the restrained muscle is fixed at a constant low length, the fiber mesh of the restrained muscle exhibits strain variations along the fiber direction as the second muscle is lengthened (Fig. 4). This is reflected on the fiber direction stresses of the same muscle.

The model results show that such distributions are also present in the lengthened muscle of the adjacent pair (Fig. 5), while the isolated muscle model exhibits highly uniform stress and strain distributions. As mean fiber direction stress within the fiber mesh is considerably lower for the lengthened muscle than for the isolated muscle, the higher distal forces associated with the lengthened muscle indicate that active force generated within one muscle may be exerted at the tendon of another muscle by intermuscular myofascial force transmission. This tendency of 'focusing' of active forces of adjacent muscles on the distal tendon of a distally lengthened muscle is a remarkable conclusion for which further experimental confirmation will be sought in the anterior tibial compartment muscles of rat hind limb.

\section{CONCLUSION}

Present application of the linked fiber matrix mesh model reveals the principles of the significant role of intermuscular myofascial force transmission on the mechanical behavior of a muscle in an in vivo situation. In agreement with experimental findings, it clearly demonstrates the altered length force characteristics with respect to the isolated situation and increases the understanding of the in vivo mechanics. The conclusion that active force generated within one muscle may be exerted at the tendon of another muscle by intermuscular myofascial force transmission is one of the remarkable results of this analysis.

\section{REFERENCES}

[1] Street, S.F., "Lateral transmission of tension in frog myofibers: a myofibrillar network and transverse cytoskeletal connections are possible transmitters," $J$ Cell Physiol, vol. 114, pp. 346-64, 1983.

[2] Hijikata, T., H. Wakisaka, and S. Niida, "Functional combination of tapering profiles and overlapping arrangements in nonspanning skeletal muscle fibers terminating intrafascicularly," Anatomical Record, vol. 236, pp. $602-610,1993$.
[3] Trotter, J.A. and P.P. Purslow, "Functional morphology of the endomysium in series fibered muscles," Journal of Morphology, vol. 212, pp. 109-22, 1992.

[4] Huijing, P.A., G.C. Baan, and G. Rebel, "Non myotendinous force transmission in rat extensor digitorum longus muscle," Journal of Experimental Biology, vol. 201, pp. 682-691, 1998.

[5] Huijing, P.A., "Muscle as a collagen fiber reinforced composite material: Force transmission in muscle and whole limbs," Journal of Biomechanics, vol. 32, pp. 329-345, 1999.

[6] Huijing, P.A., "Muscular force transmission: A unified, dual or multiple system? A review and some explorative experimental results," Archives of Physiology and Biochemistry, vol. 170, pp. 292-311, 1999.

[7] Huijing, P.A. and G.C. Baan, "Myofascial force transmission causes interaction between adjacent muscles and connective tissue: Effects of blunt dissection and compartmental fasciotomy on length force characteristics of rat extensor digitorum longus muscle," Archives of Physiology and Biochemistry, 2001, in press.

[8] Maas, H., G.C. Baan, and P.A. Huijing, "Intermuscular interaction via myofascial force transmission: Effects of tibialis anterior and extensor digitorum longus length on force transmission from rat extensor digitorum longus muscle," Journal of Biomechanics,, 2001, in press.

[9] Yucesoy, C.A., H.J.F.M. Koopman, P.A. Huijing, and H.J. Grootenboer; "Three-dimensional finite element modeling of skeletal muscle using a two-domain approach: Linked fiber-matrix mesh model,", unpublished.

[10] Danowski, B.A., K. Imanaka-Yoshida, J.M. Sanger, and J.W. Sanger, "Costameres are sites of force transmission to the substratum in adult rat cardiomyocytes," $J$ Cell Biol, vol. 118, pp. 1411-20, 1992.

[11] Huijing, P.A., "In vivo, force is transmitted from muscle also at other locations than the tendons: Extramuscular myofascial force transmisison," in Proceedings 5th annual congress of the European College of Sports Science, J. Avela, P.V. Komi, and J. Komulainen, Editors. 2000, University of Jyvaskyla: Jyvaskyla, Finland. p. 54.

[12] Faulkner, J.A., K.K. McCully, D.S. Carlson, and J.A. McNamara, Jr., "Contractile properties of the muscles of mastication of rhesus monkeys (Macaca mulatta) following increase in muscle length," Arch Oral Biol, vol. 27, pp. 841$5,1982$.

[13] Huijing, P.A., S.M. Nieberg, E.A. vd Veen, and G.J. Ettema, "A comparison of rat extensor digitorum longus and gastrocnemius medialis muscle architecture and length-force characteristics," Acta Anat, vol. 149, pp. 111-20, 1994.

[14] Jaspers, R.T., R. Brunner, J.J.M. Pel, and P.A. Huijing, "Acute effects of intramuscular aponeurotomy on rat GM: Force transmission, muscle force and sarcomere length," Journal of Biomechanics, vol. 32, pp. 71-79, 1999. 